\title{
Novice Shooters With Lower Pre-shooting Alpha Power Have Better Performance During Competition in a Virtual Reality Scenario
}

\author{
Michael Pereira ${ }^{1 \dagger}$, Ferran Argelaguet ${ }^{2 \dagger}$, José del R. Millán ${ }^{1 * \neq}$ and Anatole Lécuyer ${ }^{2 \ddagger}$ \\ ${ }^{1}$ École Polytechnique Fédérale de Lausanne, Defitech Chair in Brain-Machine Interface, Swiss Federal Institute of Lausanne, \\ Geneva, Switzerland, ${ }^{2}$ Inria, Campus de Beaulieu, Rennes, France
}

\section{OPEN ACCESS}

Edited by:

Maurizio Bertollo,

Università degli Studi G. d'Annunzio

Chieti e Pescara, Italy

Reviewed by:

Tsung-Min Hung,

National Taiwan Normal University,

Taiwan

Andrew Cooke

Bangor University, United Kingdom

*Correspondence:

José del R. Millán

jose.millan@epfl.ch

tThese authors have contributed equally to this work.

¥These authors have contributed equally to this work

Specialty section

This article was submitted to Movement Science and Sport

Psychology,

a section of the journal

Frontiers in Psychology

Received: 22 January 2018

Accepted: 27 March 2018

Published: 12 April 2018

Citation:

Pereira M, Argelaguet F, Millán JdR and Lécuyer A (2018) Novice Shooters With Lower Pre-shooting Alpha Power

Have Better Performance During

Competition in a Virtual Reality Scenario. Front. Psychol. 9:527. doi: 10.3389/fpsyg.2018.00527
Competition changes the environment for athletes. The difficulty of training for such stressful events can lead to the well-known effect of "choking" under pressure, which prevents athletes from performing at their best level. To study the effect of competition on the human brain, we recorded pilot electroencephalography (EEG) data while novice shooters were immersed in a realistic virtual environment representing a shooting range. We found a differential between-subject effect of competition on mu (8-12 $\mathrm{Hz}$ ) oscillatory activity during aiming; compared to training, the more the subject was able to desynchronize his mu rhythm during competition, the better was his shooting performance. Because this differential effect could not be explained by differences in simple measures of the kinematics and muscular activity, nor by the effect of competition or shooting performance per se, we interpret our results as evidence that mu desynchronization has a positive effect on performance during competition.

Keywords: sports, attention, EEG, virtual reality, mu, alpha

\section{INTRODUCTION}

Experimental research in Virtual Reality (VR) has a lot of potential in different research fields such as experimental brain research, psychology (McClernon et al., 2011) and sports (Wellner et al., 2010). Among its advantages, VR minimizes disturbances in complex experiments involving multiple subjects or intricate experimental protocols. In particular, VR simulation enables the accurate control and synchronization of all the elements involved in the experiment and ensures reproducibility and comparison among the different trials. In addition, due to increased user immersion and presence in the virtual environment (Slater, 2009), the engagement and the motivation of the user can be ensured.

In sport training scenarios, which are the main focus of the study presented in this paper, the VR simulation allows to immerse athletes in controlled and realistic virtual environments reproducing a competitive scenario (for instance in this study: a shooting range, competitors, and an audience) enabling the study of behavioral and physiological entangled processes (Argelaguet et al., 2015). Competition can have various effects on athletes' performances. One such effect is "choking," when athletes fail under pressure due to a stressing competitive environment. On the other hand, competition can have beneficial effects, such as a "clutch" performance, when athletes perform overly well under pressure (Ehrlenspiel, 2006). 
Electrophysiological activity in the $10 \mathrm{~Hz} \mathrm{mu/alpha}$ frequency range prior to pistol shots (Del Percio et al., 2009; Bertollo et al., 2016) or golf putts (Cooke et al., 2014) was shown to be related to performance and to dissociate experts from novices. However, conflicting evidence has been reported on the effects of competition on $\mathrm{mu}$ power, some studies finding reduced $\mathrm{mu}$ during competition (Hatfield et al., 2013) while others failing to find any effect (Cooke et al., 2014).

In this pilot study, we record novice shooters while using VR to induce a competitive shooting environment and explore both positive and negative effect of competition on the performance of novice shooters and the relation of these effects to mu power. While novice shooters are not the ultimate target population, they are arguably more subject to mild choking during controlled experiments than trained athletes.

\section{METHODS}

\subsection{Subjects}

Nine healthy subjects (age: $25.6 \pm 0.87$ mean \pm standard error of the mean) volunteered to participate in this study. Subjects reported no neurological or psychiatric problems and had normal or corrected-to-normal vision. The study was designed in accordance to the declaration of Helsinki, data was anonymized and all subjects provided prior written informed consent. Due to the minimal risk for subjects, at the time of data collection, no explicit approval form an ethical committee was sought. All subjects were right-handed. Data from one subject had to be discarded due to technical problems.

\subsection{Virtual Reality Environment}

The experiment was run in a four-sided immersive projection room with retro-projected glass screens. The dimensions of the immersive room were $9.6 \times 3.1 \times 3.1 \mathrm{~m}$ located at the Inria/IRISA laboratory at Rennes, France. The subject, head, hand position and orientation were tracked by 16 ART Tracking infrared cameras. The entire system was driven by a cluster of 14 workstations. The virtual environment reproduced a $10 \mathrm{~m}$ Olympic shooting environment in which participants had to aim and shoot at a $17 \times 17 \mathrm{~cm}$ target from a $10 \mathrm{~m}$ distance. The virtual range had seven lanes which allowed to add six virtual characters. The virtual shooting range was $9.6 \mathrm{~m}$ wide which matched the size of the CAVE (see Figure 1). Additional details regarding the virtual shooting range, the avatar behavior and behavior of participant can be found in Argelaguet et al. (2015).

\subsection{Experimental Protocol}

After reading and signing the consent form, subjects performed 2 min of eyes-opened resting state recording (baseline).

The experimental task consisted of aiming and shooting at a 17 $\times 17 \mathrm{~cm}$ target ( $0-10$ points) placed at $10 \mathrm{~m}$. Each aiming period started when the subject raised the gun with an aiming accuracy of $<2^{\circ}$. Then the subjects were instructed to aim at least $5 \mathrm{~s}$ before making a shot by gently pulling the trigger. Aiming was facilitated by displaying a virtual laser pointer and instructions were provided to ensure that all participants followed the exact same protocol.
Accuracy feedback (0-10) was provided to the subjects after each shot based on the distance between the shot and the center of the target.

Participants performed blocks of 10 shots. In each block, subjects were either alone in the shooting range (training condition), or competing against six virtual avatars with unsupportive crowd noises (competition condition).

Participants had to perform 80 shots in total, with a pause in the middle in order to rest their arm until they were ready to continue. Half of the subjects did two training blocks, followed by four competition blocks and finally two training blocks. The other half performed the experiment in the reversed order. The experiment lasted approximately $30 \mathrm{~min}$ in total.

\subsection{Electrophysiological recordings}

We recorded high-density electroencephalography (EEG) at $2,048 \mathrm{~Hz}$ in an extended 10-20 system with a Biosemi ActiveTwo amplifier. Data were downsampled to $512 \mathrm{~Hz}$ using the OpenViBE framework (Renard et al., 2010). The signals were then re-referenced (offline) to a common average. Electrooculographic (EOG) activity was measured using three additional sensors placed above the nasion and below the outer canthi of the eyes. Horizontal EOG was defined as the difference between the left and right outer canthi signals while vertical EOG was defined as the difference between the nasion and the mean of the two outer canthi signals. EOG signals were highpass filtered (zero-phase Butterworth filter, cutoff: $1 \mathrm{~Hz}$ ). Finally, bipolar electromyographic (EMG) activity was recorded over the flexor digitorum, highpass filtered (Butterworth, cutoff: $60 \mathrm{~Hz}$ ) and the envelope was computed using a Hilbert transform.

Aiming epochs were defined as the $5 \mathrm{~s}$ of data preceding a trigger pull. The 2 min eyes open baseline constituted the resting state data. For each aiming epoch and the resting state data, we computed the Welch spectrum by averaging the power of fast Fourier transform (FFT) spectra from 50\% overlapping 500 $\mathrm{ms}$ windows. The mean of each window was subtracted prior to applying the FFT to remove slow drifts of the EEG signal. Windows were eliminated from the average if the maximum amplitude difference of either the horizontal or vertical EOG was higher than $70 \mu \mathrm{V}$. Epochs with more than 50\% of EOGcontaminated windows were discarded from further analysis.

\section{RESULTS}

On a range from 1 to 7 ( 1 not at all, 7 a lot), subjects reported being competitive $(5.94 \pm 0.33)$, having medium VR experience $(4.19 \pm 0.68)$ and little shooting experience $(2.38 \pm 0.53)$. Participants rated whether the sound of the public was annoying $(2.94 \pm 0.66)$ and whether their performance was influenced by scores of avatars $(4.19 \pm 0.46)$. Mean shooting scores did not differ (non-parametric sign test, $p=0.73$ ) between training $(7.02 \pm 0.25)$ and competition $(7.14 \pm 0.26)$.

For the electrophysiological analysis, we chose (a-priori) to focus our analysis on the $\mathrm{C} 3$ electrode as it overlies the hand representation of the sensorimotor cortex. The taskrelated desynchronization was indexed as the decrease in cortical oscillatory power before the trigger pull (in $\mathrm{dB}$ ). Compared to 

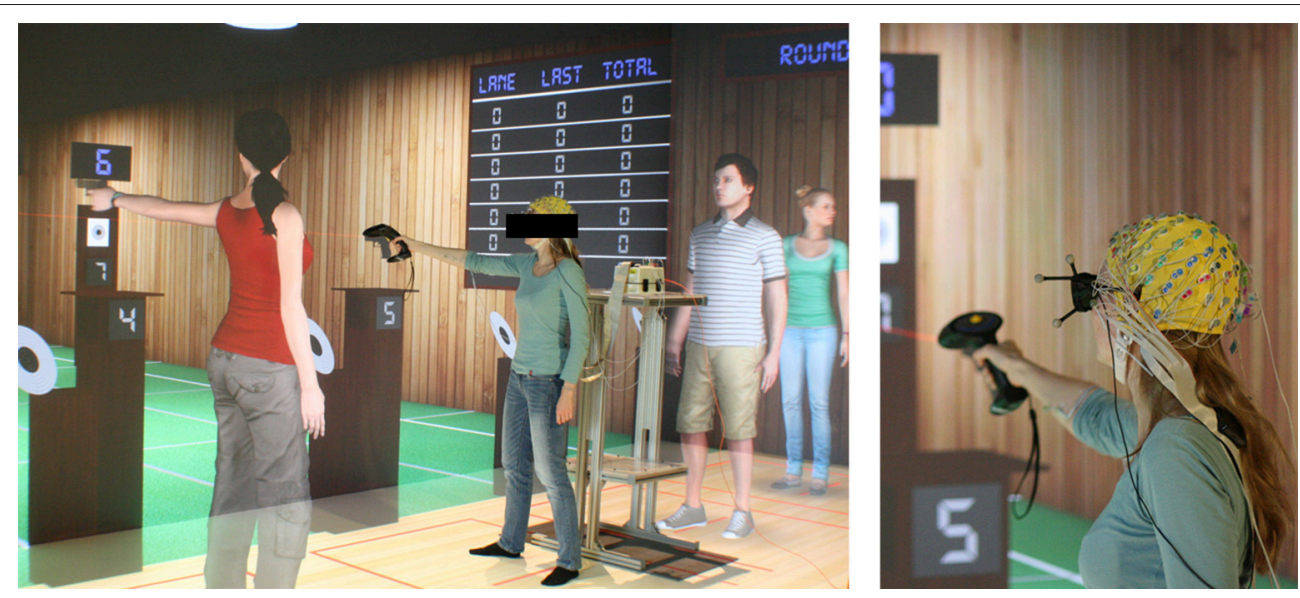

FIGURE 1 | Experimental Setup. (Left) Subjects were standing in the immersive projection system and were able to interact with the system using an ART Flystick. (Right) Subjects were wearing a high-density 64 channels EEG cap.

\section{A Spectral power increase}

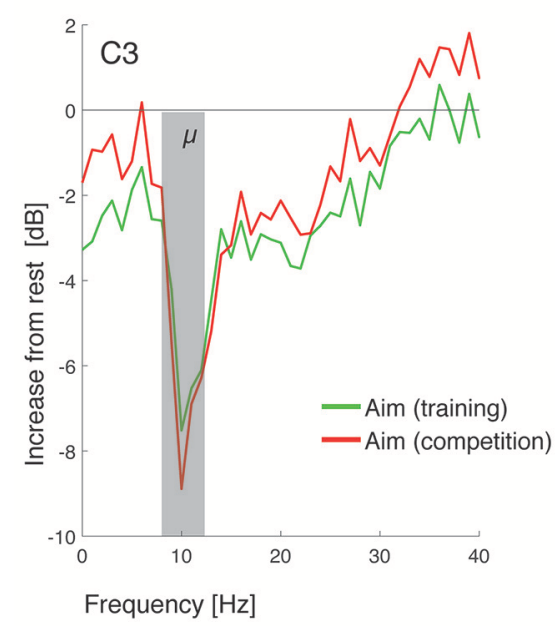

\section{B $\mu$ power difference}
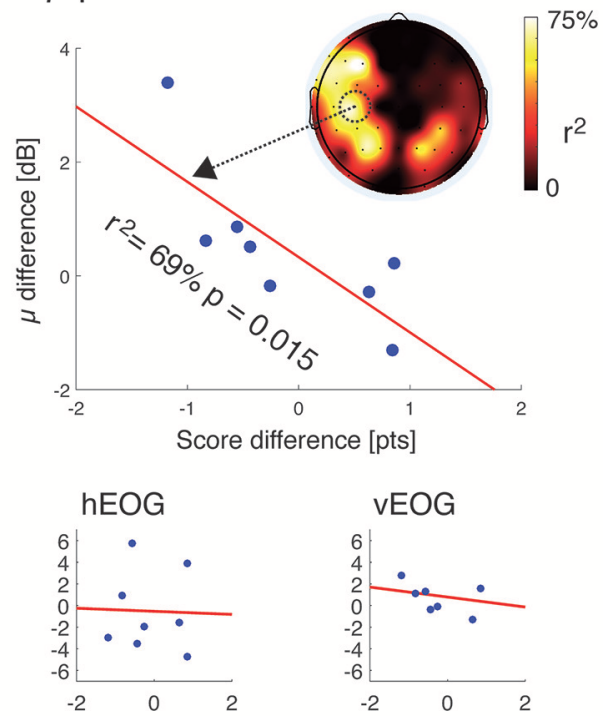

FIGURE 2 | Experimental results: (A) Desynchronization of sensorimotor rhythm during shooting compared to resting was maximum in the mu (8-12 Hz) frequency band. (B) Differential effect of competition on score and mu power correlation over electrode C3. The topographic scalp distribution of the correlation is shown on the upper right corner. Repetition of the procedure using horizontal (bottom-left) and vertical (bottom-right) EOG showed no significant correlation.

resting state data, this desynchronization was maximal at $10 \mathrm{~Hz}$ (Figure 2A).

To compare mu power between the training and the competition conditions, we averaged frequencies between 8 and $12 \mathrm{~Hz}$ (mu band). No statistically significant difference was found (non-parametric sign test, $p=0.73)$ between training $(-5.39 \pm$ $1.50[\mathrm{~dB}])$ and competition $(-2.07 \pm 1.34[\mathrm{~dB}])$. Next, we sought to find a possible differential effect, i.e., whether the effect of competition on the electrophysiology would correlate with the effect of competition on behavioral performance (score). For this, we regressed the mu difference (competition-training) from the score difference (competition-training). A significant $(p=0.015)$ portion $(69.4 \%)$ of the variance of the mu differences could be explained by the differences in score. This fitting was highest for the C3 electrode and more generally over the left sensorimotor area as well as the left parieto-occipital region (Figure 2B). Additionally, a control analysis performed on the horizontal and vertical EOG signals did not show any significant fitting $\left(r^{2}=0.2\right.$ and $0.9 \%, p=0.93$ and 0.84 , respectively), precluding this effect from being due to ocular artifacts (Figure 2B).

To verify whether our results were not simply due to higher desynchronization during good shots, we also separated good (above median performance) from bad (below median performance) shots for every subject and found no differences 
between good and bad shots (non-parametric sign test, $p=$ 0.29). Since the mu rhythm is a correlate of movement, we also verified whether muscular activity or kinematics differences could explain mu differences and repeated the analysis using EMG power differences (recorded over the flexor digitorum) and average speed of the arm (indexing lack of arm stability). Neither correlated significantly with mu differences $\left(r^{2}=8.1 \%, p=0.50\right.$ and $r^{2}=16 \%, p=0.33$, respectively).

\section{DISCUSSION}

We investigated effects of competition in shooting on $\mathrm{mu}$ frequency over the motor cortex. We used virtual reality to immerse novice shooters in a realistic shooting environment. Subjects were either alone in the shooting range or competing against six virtual avatars with loud background noises from a (virtual) unsupportive audience.

Contrarily to previous reports, we did not find consistent differences in $\mathrm{mu}$ desynchronization for competition at the group level (Hatfield et al., 2013), nor did we find an effect for good versus bad shots (Del Percio et al., 2009), which could be due to the small sample size of our study. However, we found a differential effect on mu desynchronization; subjects whose performances were impeded by the competition condition compared to when shooting alone showed lower mu desynchronization during the aiming period preceding the trigger pull (compared to when aiming alone). On the contrary, subjects for whom the competition had a positive effect on shooting performances showed higher aim-related mu desynchronization. The scalp topography of this effect, peaking over the sensorimotor area contralateral to the shooting hand is consistent with the involvement of mu desynchronization in movement preparation and execution (Pfurtscheller and Aranibar, 1979; Pfurtscheller and Lopes, 1999; Cheyne, 2013). Interestingly, the effect was also significant over the bilateral parietal areas.

We interpret the results of this pilot study as evidence that increased $\mathrm{mu}$ desynchronization in the visuomotor loop can help subjects inhibiting task-unrelated stimuli competing for attention (see Klimesch et al., 2007) and have positive effect on performance. Importantly, the differential effect of competition found in this study could not be explained by differences in

\section{REFERENCES}

Argelaguet, F., Multon, F., and Lécuyer, A. (2015). A methodology for introducing competitive anxiety and pressure in VR sports training. Front. Robot. AI 2:10. doi: 10.3389/frobt.2015.00010

Bertollo, M., di Fronso, S., Filho, E., Conforto, S., Schmid, M., Bortoli, L., et al. (2016). Proficient brain for optimal performance: the MAP model perspective. PeerJ 4:e2082. doi: 10.7717/peerj.2082

Cheyne, D. O. (2013) MEG studies of sensorimotor rhythms: a review. Exp. Neurol. 245, 27-39. doi: 10.1016/j.expneurol.2012. 08.030

Cooke, A., Kavussanu, M., Gallicchio, G., Willoughby, A., Mcintyre, D., and Ring, C. (2014). Preparation for action: Psychophysiological activity movement instabilities. It remains to show whether this effect can be generalized to expert shooters.

Our findings could be relevant in sports training to help athletes avoid choking under pressure during competition. Confirmation through further experimental validation is needed.

\section{ETHICS STATEMENT}

There was no explicit approval by an ethical committee for our study. However, please note that:

1) All subjects participating in the experiment gave written informed consent in accordance with the Declaration of Helsinki. In particular, the informed consent form described the experimental protocol, detailed the rights and obligations for the subjects, described any potential risks and asked explicitly their consent regarding the use of the data gathered during the experiment.

2) Due to the minimal risk in the experiment for the subjects (physically and mentally), the fact that data recording was anonymized, and that we were not dealing with sensible data, we considered that there was no need for an explicit approval of the ethical committee. Indeed, at the moment of the experiment (end of 2014), the institution where experiments took place (Computer Science background) didn't have the culture of collecting systematically an explicit approval of the ethical committee for this kind of experiments -this requirement has clearly changed over the last years.

\section{AUTHOR CONTRIBUTIONS}

MP and FA contributed equally. AL and JM also contributed equally. All authors designed the experiment and wrote the paper. MP and FA collected data. FA developed the VR framework. MP analyzed the EEG data. JM and AL oversaw the project.

\section{ACKNOWLEDGMENTS}

This work was partially supported by the Vision Advanced Infrastructure for Research (VISIONAIR) FP7 Project, with the support of the Immersia Platform at Inria Rennes. In addition, we would like to thank Jussi T. Lindgren for the OpenViBE support and reviewing the manuscript.

preceding a motor skill as a function of expertise, performance outcome, and psychological pressure. Psychophysiology 51, 374-384. doi: 10.1111/psyp. 12182

Del Percio, C., Babiloni, C., Bertollo, M., Marzano, N., Iacoboni, M., Infarinato, F., et al. (2009). Visuo-attentional and sensorimotor alpha rhythms are related to visuo-motor performance in athletes. Hum. Brain Mapp. 30, 3527-3540. doi: 10.1002/hbm. 20776

Ehrlenspiel, F. (2006). Chocking Under Pressure-Attention and Motor Control in Performance Situations. Ph.D. thesis, University of Potsdam.

Hatfield, B. D., Costanzo, M. E., Goodman, R. N., Lo, L. C., Oh, H., Rietschel, J. C., et al. (2013). The influence of social evaluation on cerebral cortical activity and motor performance: a study of "Real-Life" 
competition. Int. J. Psychophysiol. 90, 240-249. doi: 10.1016/j.ijpsycho.2013. 08.002

Klimesch, W., Sauseng, P., and Hanslmayr, S. (2007). EEG alpha oscillations: the inhibition-timing hypothesis. Brain Res. Rev. 53, 63-88. doi: 10.1016/j.brainresrev.2006.06.003

McClernon, C. K., McCauley, M. E., O'Connor, P. E., and Warm, J. S. (2011). Stress training improves performance during a stressful flight. Hum. Factors 53, 207-218. doi: 10.1177/0018720811405317

Pfurtscheller, G., and Aranibar, A. (1979). Evaluation of event-related desynchronization (ERD) preceding and following voluntary self-paced movement. Electroencephalogr. Clin. Neurophysiol. 46, 138-146.

Pfurtscheller, G., and Lopes, F. H. (1999). Event-related EEG/MEG synchronization and desynchronization : basic principles. Clin. Neurophysiol. 110, 1842-1857.

Renard, Y., Lotte, F., Gibert, G., Congedo, M., Maby, E., Delannoy, V., et al. (2010). OpenViBE: an Open-Source software platform to design, test, and use Brain Computer Interfaces in real and virtual environments. Neural Comput. 19, 35-53. doi: 10.1162/pres.19.1.35
Slater, M. (2009). Place illusion and plausibility can lead to realistic behaviour in immersive virtual environments. Philos. Trans. R. Soc. Lond. B Biol. Sci. 364, 3549-3557. doi: 10.1098/rstb.2009.0138

Wellner, M., Sigrist, R., von Zitzewitz, J., Wolf, P., and Riener, R. (2010). Does a virtual audience influence rowing? Proc. Inst. Mech. Eng. 224, 117-128. doi: 10.1243/17543371JSET33

Conflict of Interest Statement: The authors declare that the research was conducted in the absence of any commercial or financial relationships that could be construed as a potential conflict of interest.

Copyright (c) 2018 Pereira, Argelaguet, Millán and Lécuyer. This is an open-access article distributed under the terms of the Creative Commons Attribution License (CC $B Y)$. The use, distribution or reproduction in other forums is permitted, provided the original author(s) and the copyright owner are credited and that the original publication in this journal is cited, in accordance with accepted academic practice. No use, distribution or reproduction is permitted which does not comply with these terms. 